\title{
When Should Congenital Inherited Thrombocytopenia Be Suspected?
}

\author{
a report by \\ JF Viallard and $\mathbf{P}$ Nurden
}

French National Reference Centre for Inherited Platelet Diseases

DOI: 10.17925/EOH.2007.0.0.23

In the case of an isolated chronic thrombocytopenia in an adult, the diagnostic procedure is well established. First, several causes must be ruled out. These include:

- false thrombocytopenia diagnosis caused by platelet aggregation and clumping in the presence of ethylenediaminetetraacetic acid (EDTA) anticoagulant;

- haematological malignancy and myelodysplastic syndromes;

- viral infections (particularly HIV infection);

- autoimmune diseases such as systemic lupus erythematosus (SLE) and/or antiphospholipid syndrome;

- disseminated intravascular coagulopathy;

- thrombotic thrombocytopenic purpura; and

- splenic sequestration.

When these causes have been excluded, an antibody-mediated mechanism is usually suspected and a diagnosis of immune thrombocytopenic purpura (ITP) proposed. However, since Nurden and Caen first identified the molecular defect in patients with Bernard-Soulier syndrome in 1975, ${ }^{1}$ the genetic causes of a growing number of congenital thrombocytopenias (CTPS) have been elucidated, a number of cases of CTP have been misdiagnosed as ITP and the patients have been subjected to splenectomy and/or cyclophosphamide, among other inappropriate therapies.

Recent developments, especially in the identification of molecular defects, have highlighted the characteristic features of congenital cases of non-immune thrombocytopenia and improved our ability to identify them. ${ }^{2}$ However, the specialised testing needed to diagnose platelet disorders is often restricted to specialised centres. The challenge for the physician is to identify patients who will benefit from specific investigation procedures. The aim of this article is to assist clinicians in distinguishing between genetic and immune thrombocytopenia. There are at least four criteria that suggest CTP. The first is a family history of thrombocytopenia; the second, the absence of an increase in the platelet count in response to ITP treatments; the third, the presence of special associated features; and the fourth, an abnormal platelet size assessed by examination of a peripheral blood smear.

\section{Familial History of Thrombocytopenia}

While non-specific, the most obvious feature suggesting CTP is the presence of a family history of thrombocytopenia. This should immediately raise suspicion of CTP rather than ITP, especially if more than two family members are involved and/or the family members are closely related. Nevertheless, a family history of ITP has also been reported in familial autoimmune disease in which the family history may be more consistent with SLE. ${ }^{3}$
A family history of leukaemia suggests a familial thrombocytopenialeukaemia (Tel-AML1) syndrome. It is important to indentify this as it is the thrombocytopenia most often linked to malignancy. ${ }^{4}$ Approximately half of patients go on to develop a malignancy, two-thirds of which are myeloid leukaemias and one-third of which are solid tumours. Recently, mutations in the AML1 (Runx1 or CBFA2) gene have been identified in affected members of families with autosomal dominant thrombocytopenia and abnormal platelet functions. AML1 encodes a transcription factor that is widely expressed in multiple haematopoietic lineages and that regulates the expression of a variety of haematopoietic genes. AML1 is particularly critical for megakaryopoiesis and is a frequent target for chromosomal translocation in human leukaemia. Many of the patients affected, usually by point mutations in one allele, are predisposed to the development of acute myeloid leukaemia in adult life. ${ }^{5}$

\section{Response to Idiopathic Thrombocytopenic Purpura Treatments}

Another suggestive indicator for CTP is the 'failure' to respond to ITP-specific therapy. This includes not only the use of intravenous immunoglobulins (IVIgs), steroids or other immune-modulating therapies such as azathioprine, cyclophosphamide and rituximab, but also splenectomy. While the lack of response in patients with CTP to these treatments is a universally accepted criterion based on anecdotal experience, an exact definition of lack of response has not been established. For example, how much does the platelet count have to increase by after treatment with IVIg to diagnose ITP and exclude CTP? Arbitrarily, a peak platelet response to treatment $>30,000 / \mu$ increase from baseline would suggest that the case in question is not CTP. Conversely, $\mathrm{a}<10,000 / \mu \mathrm{l}$ peak increase is compatible with CTP, although it is just as compatible with a diagnosis of 'refractory' ITP. 6 Numbers in between might favour ITP, but are even more ambiguous.

A confusing feature is that some patients with CTP (with Wiskott-Aldrich syndrome, for example) respond to corticosteroids, IVIg and/or splenectomy either because there is an immunological component to the thrombocytopenia or because impeding the clearance mechanism for aberrant platelets helps to offset impaired platelet production. As 'spontaneous' fluctuation in the platelet count may occur (for example as a result of a viral infection), the assessment of two treatment responses is probably more helpful as a diagnostic criterion.

\section{Associated Features}

Some features, when identified in patients with persistent thrombocytopenia, suggest specific types of CTP. For example, bilateral radial aplasia (often associated with other orthopaedic abnormalities of the ulna and knees) is suggestive of thrombocytopenia with absent radii (TAR) syndrome. ${ }^{7}$ Patients with TAR have a high incidence of serious 
bleeding, including intracranical haemorrhage $(\mathrm{ICH})$ and gastrointestinal (GI) bleeding. However, these patients tend to improve their platelet counts with time. The dogma is that these patients will achieve normal platelet counts within one year of birth. However, milder thrombocytopenia often persists and platelet levels may fall again during adulthood. Signalling via the thrombopoietin receptor is abnormal, but the defect in the signalling pathway has not been defined.

Patients with velocardiofacial (VCF) syndrome and DiGeorge syndrome, marked by cardiac abnormalities, parathyroid and thymus insufficiencies, cognitive/learning impairment and facial dysmorphology (VCF only) have deletions within human chromosome 22q11 and are collectively referred to as cardiac abnormality, T cell deficit, cleft palate and hypocalcaemia due to Chr22 deletion ('CATCH22') patients. ${ }^{8}$

Patients with either Wiskott-Aldrich syndrome (WAS) or the X-linked thrombocytopenia (XLT) form of WAS usually have marked or severe thrombocytopenia and platelets that are smaller than normal. ${ }^{9}$ In addition to severe thrombocytopenia, WAS is a significant congenital immunodeficiency syndrome that includes an inability to produce antipolysaccharide antibodies, resulting in a predilection to pneumococcal sepsis. Eczema is common and newborns and very young infants may present with a milk allergy and haematochezia. However, it is important to note that frequent infections may overlap with immune thrombocytopenias secondary to hypogammaglobulinaemia. ${ }^{10}$

High-tone hearing loss, renal failure and cataracts are suggestive of myosin heavy chain 9 (MYH9)-related macrothrombocytopenia syndromes. ${ }^{11}$ Syndromes in this category include the May-Hegglin anomaly, Fechtner syndrome, Sebastian syndrome and Epstein syndrome. The gene mutated in all four of these autosomal dominant macrothrombocytopenias was mapped to the chromosome 22q12-13, and has recently been identified as MYH9, encoding the non-muscle myosin heavy-chain IIA (NMMHC-IIA).

Assessment of the Platelet Size on Peripheral Blood Smear As many of the CTPs have abnormal platelet morphology, careful examination of the peripheral blood smear should be performed for all patients with newly diagnosed thrombocytopenia. Newer automatic blood cell analysers are superior to the previous generation in their recognition of platelets; nevertheless, the larger platelets are not recognised, so the mean platelet volume obtained in standard laboratories does not reflect the whole platelet population. The visual inspection of the smear remains the 'gold standard' for platelet size in clinical practice. The recognition of giant platelets or microthrombocytes helps to narrow the diagnostic possibilities. For example:
- if platelets appear very large (the size of red cells or even larger), this is compatible with Bernard-Soulier syndrome or the MYH9 defects;

- the presence of very small platelets is most consistent with Wiskott-Aldrich syndrome, whether the complete syndrome or the XLT form; and

- platelet clumping may suggest von Willebrand type $\mathrm{llb}$, although pseudothrombocytopenia would need to be excluded either by using citrate as the anticoagulant or by making smears directly from a drop of blood.

Microscopic examination of blood smears with May-Grünwald-Giemsa staining can also reveal light-blue leukocyte inclusions called Döhle-like bodies in the cytoplasm of neutrophils, suggesting May-Hegglin syndrome. In fact, while the NMMHC-IIA is homogeneously distributed within granulocyte cytoplasm, it always presents a spotty distribution in MYH9-related diseases, and inclusion leukocytes correspond to these spots. Immunocytochemical studies with antibodies directed against NMMHC-IIA represent a more sensitive method of detecting the irregular distribution of myosin. ${ }^{11}$ A grey (uncoloured) appearance will signal the Grey platelet syndrome, where thrombocytopenia is associated with a lack of $\alpha$-granules.

\section{Other Abnormalities Suggesting Congenital Thrombocytopenia}

There are other clinical characteristics for recognising inherited thrombocytopenia, but they are non-specific and have little predictive value. Bleeding is not an important criterion as a large group of individuals with inherited thrombocytopenia may never have clinically significant spontaneous bleeding and will be identified during routine blood tests with mild/moderate thrombocytopenia (e.g. $>20,000 / \mu l$ ). However, any discordance between the severity and frequency of bleeding and a relatively high platelet count is probably suggestive of a congenital functional defect. In the same way, when there is doubt as to the reality of the immune mechanism, the determination of the platelet lifespan by isotopic study can help the clinician. Classically, reduced platelet lifespan is suggestive of ITP. Nevertheless, it has recently been shown in an animal model that deletion of the $\mathrm{Bcl}-\mathrm{x}$ gene, involved in apoptosis, induces a shortened platelet lifespan.12

\section{Conclusion}

Despite careful clinical and biological analysis, we have to admit that a substantial number of patients will not fit into any of the disorders described. In these cases it is reasonable to refer these patients to a haematologist specialised in inherited platelet disorders. Future research will be focused on the analysis of the megakaryocyte lineage in these patients. In the same way, the focus will now turn towards new rational therapies, for example the use of new agonists of the thrombopoietin receptor.
1. Nurden AT, Caen JP, Specific roles for platelet surface glycoproteins in platelet function, Nature, 1975;255:720-22.

2. Hayward CPM, Inherited platelet disorders, Curr Opin Hematol, 2003;10:362-8.

3. Scofield RH, Bruner GR, Kelly JA, et al., Thrombocytopenia identifies a severe familial phenotype of systemic lupus erythematosus and reveals genetic linkages at $1 \mathrm{q} 22$ and $11 \mathrm{p} 1$ Blood, 2003;101:992-7.

4. Loh ML, Rubnitz JE, TEL/AML1-positive paediatric leukaemia: prognostic significance and therapeutic approaches, Curr Opin Hematol, 2002;9345-52.

5. Song WJ, Sullivan MG, Legare RD, al., Haploinsufficiency of CBFA2 causes familial thrombocytopenia with propensity to develop acute myelogenous leukaemia, Nat Genet, 1999;23:166-75.

6. Cines DB, Bussel JB, McMillan RB, Zehnder JL, Congenital and acquired thrombocytopenia, Hematology Am Soc Hematol Educ Program, 2004;390-406.

7. Geddis $A E$, Inherited thrombocytopenia: Congenital amegakaryocytic thrombocytopenia and thrombocytopenia with absent radii, Semin Hematol, 2006;43:196-203.

8. Ryan AK, Goodship JA, Wilson DI, et al., Spectrum of clinical features associated with interstitial chromosome 22q11 deletions: a European collaborative study, J Med Genet, 1997;34:798-804.

9. Ochs HD, Thrasher AJ, The Wiskott-Aldrich syndrome,
Allergy Clin Immunol, 2006;117:725-38.

10. Michel M, Chanet V, Galicier L, et al., Autoimmune thrombocytopenic purpura and common variable immunodeficiency: analysis of 21 cases and review of the literature, Medicine, 2004;83:254-63.

11. Seri M, Pecci A, Di Bari F, Cusano R, et al., MYH9-related disease: May-Hegglin anomaly, Sebastian syndrome, Fechtner syndrome and Epstein syndrome are not distinct entities but represent a variable expression of a single illness, Medicine, 2003:82:203-15.

12. Mason KD, Carpinelli MR, Fletcher Jl, et al., Programmed anuclear cell death delimits platelet life span, Cell, 2007; 128:1173-86. 